\title{
Joint Design of Transmission Rate and Control for Wireless Sensor Networked Control Systems
}

\author{
Jinna Li, ${ }^{1,2}$ Peng Zeng, ${ }^{2}$ Xuejun Zong, ${ }^{3}$ Meng Zheng, ${ }^{2}$ and Xiaoling Zhang ${ }^{2}$ \\ ${ }^{1}$ Lab of Operation and Control, Shenyang University of Chemical Technology, Liaoning 110142, China \\ ${ }^{2}$ Key Laboratory of Networked Control Systems, Shenyang Institute of Automation, Chinese Academy of Sciences, \\ Liaoning 110016, China \\ ${ }^{3}$ College of Information Engineering, Shenyang University of Chemical Technology, Liaoning 110142, China
}

Correspondence should be addressed to Jinna Li; lijinna_721@126.com

Received 19 March 2014; Revised 11 April 2014; Accepted 13 April 2014; Published 28 May 2014

Academic Editor: Jun Cheng

Copyright (C) 2014 Jinna Li et al. This is an open access article distributed under the Creative Commons Attribution License, which permits unrestricted use, distribution, and reproduction in any medium, provided the original work is properly cited.

This paper is concerned with transmission rate and control codesign of wireless sensor networked control systems (WSNCS) with time-varying delay. Jointly designing transmission rate and control is an attractive paradigm for WSNCS, since the control performance of WSNCS is highly sensitive to resource-constrained communication networks. The main idea of devised scheme is searching an optimal event-triggered transmission condition on the premise that wireless link capacity constraint is satisfied and stability of systems is guaranteed. The main aim of devised scheme is to greatly optimize control performance of WSNCS. First, two wireless network architectures characterized by multihop and star topology are put forward. Secondly, a model of WSNCS with event-triggered transmission mechanism is constructed. It is followed by stability analysis of WSNCS to obtain the asymptotical stability condition of systems. And then a search algorithm is presented for transmission rate and control codesign. Finally, numerical examples are given to illustrate the effectiveness of the proposed method.

\section{Introduction}

Networked control systems (NCS) are distributed feedback systems wherein controlled nodes are distributed and connected over a network medium [1-5]. If the wireless sensor networks (WSN) are utilized to be media used for control systems information transfer, the special feedback control system is called wireless sensor networked control systems (WSNCS). WSN are composed of spatially distributed static or mobile nodes with sensing, processing, and transmitting functions. The topology structure of WSN includes star networks, mesh networks, and hybrid networks. In star topology networks with single-hop structure, all of the sensor nodes directly communicate with a central node (base station). Different from this type of networks, all of the sensor nodes can directly communicate with each other and each sensor node can transmit information to a base station by multihop approach. In addition, hybrid networks are characterized by both star and mesh topology. It is well known that the advantages (such as flexible installation and maintenance, lost cost) enable WSNCS to be widely used in smart grids, industrial process control, and so forth $[6,7]$. Based on the different application scenarios, one can determine what type of network topology structures is more appropriate. Star topology network architectures are highly relevant in process industry $[6,7]$, and mesh or hybrid network architecturesbased networked control has been founded in greenhouse applications [8].

However, a common issue which needs to be faced by WSNCS is resource-constrained environment. The packet loss and long or unbounded transmission delay inevitably occur when transmission rate exceeds the bandwidth constraint due to limited link capacity. However, the requirement of most of the industry control process on reliability is over $95 \%$ and smart gird requires that delay is smaller than $1 \mathrm{~ms}$ in the substations. Thus, only controller design used to overcome the negative factors, such as delay and packet loss, fails to stabilize the WSNCS. Hence, it is quite necessary 
and urgent to jointly design communication and control for WSNCS. A rapidly increasing focus on the joint design of communication and control for NCS has been witnessed [920], such as codesign of controller and capacity allocation [9], codesign of scheduling and control system [10, 11], energy-aware adaptive cooperative control [12], maximum network lifetime [13], and codesign of sampling and control systems[14-20].

In this paper, the event-triggered sampling or transmission scheme as a promising alternative for codesign of communication and control is considered as research object for WSNCS with star and mesh (multihop) network topology. The proposed codesign method can be considered as the reference for WSNCS with hybrid topology structure since they consist of star and mesh networks. Compared with time-triggered sampling scheme, whether the data should be sampled or not is verified based on a special triggering condition in event-triggered sampling policy. This approach allows a considerable reduction of network load while guaranteeing the specific control performance [14-19]. In [14-17], we easily find a common feature that controller is first designed and then an eventtriggering condition is determined. This means that the event-triggering sampling scheme for NCS is completed by two steps. By following such results, [18] designed an algorithm to derive controller gains and the event-triggering transmission condition in one step while meeting $H_{\infty}$ performance with respect to disturbance. In [19], an eventtriggering condition is derived by using passivity theory such that a large class of output feedback stabilizing controllers can be obtained. Additionally, [20] proposed a cross-layer design method to achieve both stability and performance optimization.

Note that sometimes event-triggered control might not contribute to the reduction of network resource occupancy because transmissions are enforced to go on when transmission condition is triggered to reach a desired control performance. Under the triggering condition, network even might be overloaded leading to degradation of control performance of systems. Hence, how to determine the triggering scheme under communication constraints such that the desired control performance is achieved is a key to be investigated. Although the above works on event-triggered sampling or transmitting control for NCS have recently appeared in the literature, communication constraints associated with sampling or transmission still have not been taken into account, which motivates the study presented in this paper.

In this paper, two wireless network architectures characterized by multihop and star topology are first put forward. Based on it, a model of WSNCS with event-triggered transmission mechanism is proposed. Furthermore, a sufficient condition for asymptotical stability of WSNCS is derived based on Lyapunov method combined with linear matrix inequalities (LMI) approach. And then a search algorithm to obtain the optimal triggering condition is presented for communication and control codesign. It should be pointed out that the optimal triggering condition can guarantee the optimal control performance of WSNCS on the premise that flow rate meets wireless link capacity constraint. Finally, numerical examples are given to illustrate the effectiveness of the proposed method.

The two main contributions of this paper can be summarized below.

(1) Compared with the existing results on event-triggered sampling scheme used in NCSs [14-19], the most significant advantage of the proposed method is that we consider link capacity constraint when designing transmission scheme. Only if the transmission triggering condition is satisfied, sampled data are transferred over networks. However, this condition is likely to cause a condition in which the flow rate exceeds the link capacity constraint, which is not investigated in [14-19].

(2) Similar to [20], wireless multihop sensor networks are considered as search subject when designing transmission scheme, but there are mainly two differences. One is that we present transmission rate and control codesign method for WSNCS with star topology networks besides multihop sensor networks. The other is that we adopt event-triggered scheme for saving bandwidth, while [20] obtained the optimal transmission rate by solving nonconvex optimization control based on cross-layer approach.

This paper is organized as follows. The problem statement is presented in Section 2. Section 3 is dedicated to stability analysis of WSNCS. Section 4 presents the transmission rate and control codesign scheme. Numerical simulation results are presented in Section 5. Conclusions are stated in Section 6.

Notation. $\mathfrak{R}^{n}$ and $\mathfrak{R}^{n \times m}$ denote the $n$ dimensional Euclidean space and the set of all $n \times m$ real matrices, respectively. $\mathbf{N}$ denotes the set of nonnegative integers; $\mathbf{P}>\mathbf{0}$ means that matrix $\mathbf{P}$ is real symmetric and positive definite. The subscript " $T$ " denotes the matrix transpose. $\sup (\cdot)$ denotes the supremum of $(\cdot)$. In symmetric block matrices, we use " $*$ " to represent a term that is induced by symmetry.

\section{Problem Statement}

2.1. Control System Model. Every continuous-time plant is governed by

$$
\dot{\mathbf{x}}_{i}(t)=\mathbf{A}_{i} \mathbf{x}_{i}(t)+\mathbf{B}_{i} \mathbf{u}_{i}(t)
$$

where $\mathbf{x}_{i}(t) \in \mathfrak{R}^{n}$ and $\mathbf{u}_{i}(t) \in \mathfrak{R}^{m}(i \in H)$ are the state vector and control input vector, respectively; $\mathbf{A}_{i}$ and $\mathbf{B}_{i}$ are some constant matrices of appropriate dimensions. $H=$ $\{1,2, \ldots, G\}$ represents the set of plants, and $G$ is the number of controlled plants.

The state feedback controller for each control loop can be expressed as

$$
\mathbf{u}_{i}(t)=\mathbf{K}_{i} \mathbf{x}_{i}(t)
$$

where $\mathbf{K}_{i}$ is some constant matrix of appropriate dimensions. 
2.2. Wireless Sensor Network Architectures. A multihop wireless sensor network as shown in Figure 1 is modeled as a directed graph $G=(V, L)$, where $V$ and $L$ represent the set of sensor nodes and the set of directed wireless links $l(l \in L)$, respectively [20]. For a special case in which star topology structure is applied in wireless sensor networks, information is transmitted from sensor nodes to a central node (base station). In this network, the central node is wired to the controllers; see Figure 2. Such two types of networks can be shared by control systems $H$, and we use $F(i)$ to denote the end-to-end flow generated by the traffic from the plant to controller for each $i \in H$. And $L(f)$ and $F(l)$ are used to denote all of the links that flow $f$ traverses and all of the flows that pass by the link $l$, respectively.

\subsection{Event-Triggered Networked Control System Formulation.} It is well known that the digital controller and zero-order holder $(\mathrm{ZOH})$ are usually applied in the WSNCS; in this context, the states of systems are sampled and are transmitted over networks. For every controlled plant $i(i \in H)$, we assume that the sensor is time-triggered at the time instant $i_{k} h\left(i_{k}=0,1,2, \ldots\right)$, where $k \in \mathbf{N}$. The sampling sequence is denoted by the set $\mathbf{S}_{1}=\{0, h, 2 h, \ldots\}$ and $h$ is a constant sampling interval. Moreover, we also assume transmission over network is triggered by an event. Thus the transmission sequence at the sensors is described by the set $\mathbf{S}_{i, 2}=$ $\left\{0, b_{i, 1} h, b_{i, 2} h, \ldots, b_{i, k} h, \ldots\right\}$, where $b_{i, k} \in \mathbf{N}$ and $\mathbf{S}_{i, 2} \subseteq \mathbf{S}_{1}$.

First a designed transmission scheme is proposed as follows [18]:

$$
b_{i, k+1} h=b_{i, k} h+\min _{l_{i}}\left\{l_{i} h \mid e_{i}^{T}\left(i_{k} h\right) \Phi e_{i}\left(i_{k} h\right) \geq \sigma_{i} \wp\right\},
$$

where $e_{i}\left(i_{k} h\right)=x_{i}\left(i_{k} h\right)-x_{i}\left(b_{i, k} h\right)$ denotes the error between the states at the current sampling time instant and at the latest transmitted sampling time instant, $\wp=x_{i}^{T}\left(b_{i, k} h\right) \Phi x_{i}\left(b_{i, k} h\right)$, and $\sigma_{i} \geq 0(i \in H)$ is a given scalar parameter.

Remark 1. Not all of the sampled data will be transmitted, whether or not they should be transmitted based on the transmission scheme (3). This means that $i_{k}=b_{i, k}+l_{i} h\left(l_{i} \in\right.$ $\mathbf{N})$. Hence, there exists the error between the states at the current sampling time instant and at the latest transmitted sampling time instant. Only if the error goes over the bound $\sigma_{i} \wp$, the sampled data are sent.

Then, we construct a WSNCS model based on the above event-triggered transmission scheme. A wireless sensor network is used to connect the plants (1) with the controllers (2), and the state feedback controllers are directly connected to the actuator by $\mathrm{ZOH}$, and thus (1) can be rewritten as

$$
\begin{aligned}
& \dot{\mathbf{x}}_{i}(t)=\mathbf{A}_{i} \mathbf{x}_{i}(t)+\mathbf{B}_{i} \mathbf{u}_{i}(t), \\
& \mathbf{u}_{i}(t)=\mathbf{K}_{i} \mathbf{x}_{i}\left(b_{i, k} h\right)
\end{aligned}
$$

for $t \in\left[b_{i, k} h+\tau_{i, k}, b_{i, k+1} h+\tau_{i, k+1}\right)$, where $\tau_{i, k}$ denotes the transmission delay experienced by transmitted packet at $b_{i, k} h$ time instant.

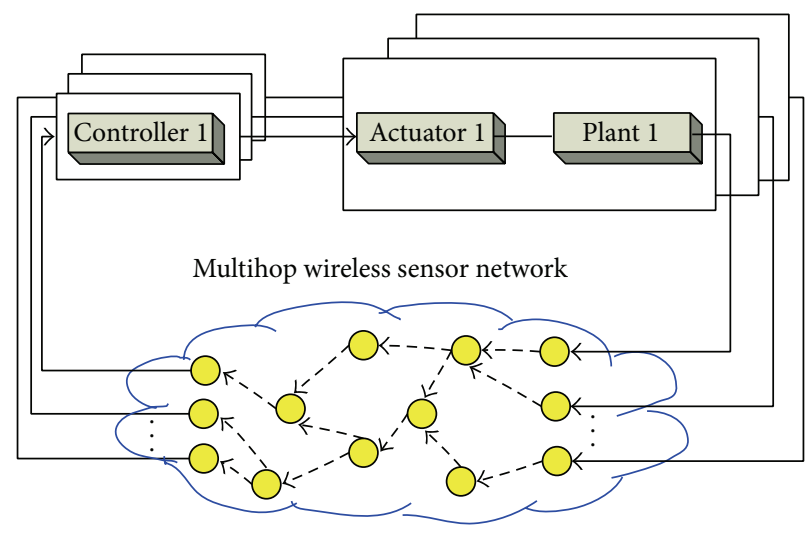

FIGURE 1: A wireless multihop sensor network.

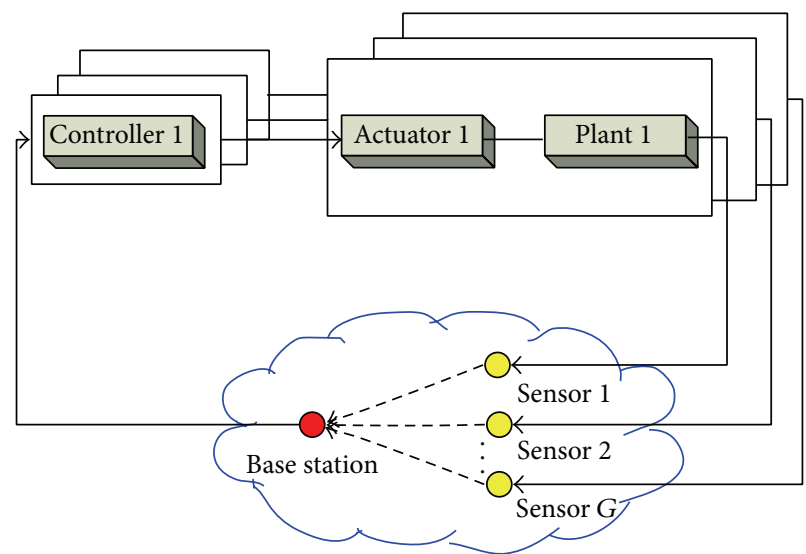

FIGURE 2: Star topology wireless sensor network.

Remark 2. In this paper, we mainly focus on how to jointly design transmission rate and control for WSNCS, so it is assumed that packet loss is absent for the simplicity. That means that the transmitted samples must be received by the plants.

Define $\eta(i, t)=t-b_{i, k} h$; it is clear that $0 \leq \tau_{i, k} \leq \eta(i, t) \leq$ $b_{i, k+1} h-b_{i, k} h+\tau_{i, k+1}$. Set $\bar{\tau}=\sup _{i, k} \tau_{i, k}$ and $\bar{b}=\sup _{i, k}\left(b_{i, k+1} h-\right.$ $\left.b_{i, k} h\right)$; then $\eta(i, t) \leq \eta=\bar{\tau}+\bar{b}$. Thus, (4) can be described as a time delay-dependent system

$$
\begin{aligned}
& \dot{\mathbf{x}}_{i}(t)=\mathbf{A}_{i} \mathbf{x}_{i}(t)+\mathbf{B}_{i} \mathbf{K}_{i} \mathbf{x}_{i}(t-\eta(i, t)), \\
& \mathbf{x}_{i}(t)=\phi_{i}(t), \quad t \in\left[t_{0}-\eta, t_{0}\right], \phi_{i}\left(t_{0}\right)=\mathbf{x}_{0},
\end{aligned}
$$

where $\phi_{i}(t)$ and $\phi_{i}\left(t_{0}\right)$ are the initial states of system $i$ during the time period $\left[t_{0}-\eta, t_{0}\right]$ and at the initial time instant $t_{0}$, respectively.

\section{Stability Analysis of WSNCS}

In this section, stability analysis is presented for the concerned WSNCS. This result is critical for transmission rate and control codesign. 
Theorem 3. For given scalars $\eta$ and $\sigma_{i}$, a matrix $\mathbf{K}_{i}$, under the transmission rule (3), the system (5) is asymptotically stable, if there exist matrices $\mathbf{N}_{i j}, \mathbf{M}_{i j}(j=1,2,3), \mathbf{P}_{i}>\mathbf{0}, \mathbf{T}_{i}>\mathbf{0}$ and $\Phi_{i}>0$, such that

$$
\left[\begin{array}{ccccc}
\Theta_{i, 11} & \Theta_{i, 12} & \Theta_{13} & \eta \mathbf{N}_{i 1} & \mathbf{0} \\
* & \Theta_{i, 22} & \Theta_{i, 23} & \eta \mathbf{N}_{i 2} & \mathbf{0} \\
* & * & \Theta_{i, 33} & \eta \mathbf{N}_{i 3} & \mathbf{0} \\
* & * & * & -\eta \mathbf{T}_{i} & \mathbf{0} \\
* & * & * & * & -\Phi_{i}
\end{array}\right]<0
$$

where

$$
\begin{aligned}
& \Theta_{i, 11}=\mathbf{N}_{i 1}+\mathbf{N}_{i 1}^{T}-\mathbf{M}_{i 1}-\mathbf{M}_{i 1}^{T}, \\
& \Theta_{i, 12}=\mathbf{N}_{i 2}^{T}-\mathbf{N}_{i 1}-\mathbf{A}_{i}^{T} \mathbf{M}_{i 2}^{T}-\mathbf{M}_{i, 1} \mathbf{B}_{i} \mathbf{K}_{i}, \\
& \Theta_{i, 13}=\mathbf{N}_{i 3}^{T}-\mathbf{A}_{i}^{T} \mathbf{M}_{i 3}^{T}+\mathbf{M}_{i 1}+\mathbf{P}, \\
& \Theta_{i, 22}=-\mathbf{N}_{i 2}-\mathbf{N}_{i 2}^{T}-\mathbf{M}_{i 2} \mathbf{B}_{i} \mathbf{K}_{i}-\mathbf{K}_{i}^{T} \mathbf{B}_{i}^{T} \mathbf{M}_{i 2}^{T}+\sigma_{i} \Phi, \\
& \Theta_{i, 23}=-\mathbf{N}_{i 3}^{T}+\mathbf{M}_{i 2}-\mathbf{K}_{i}^{T} \mathbf{B}_{i}^{T} \mathbf{M}_{i 3}^{T}, \\
& \Theta_{i, 33}=\mathbf{M}_{i 3}^{T}+\mathbf{M}_{i 3}+\eta \mathbf{T}_{i} .
\end{aligned}
$$

Proof. Choose a Lyapunov-Krasovskii functional candidate as follows [21]:

$$
V(t)=\mathbf{x}_{i}^{T}(t) \mathbf{P}_{i} \mathbf{x}_{i}(t)+\int_{t-\eta}^{t} \int_{s}^{t} \dot{\mathbf{x}}_{i}^{T}(v) \mathbf{T}_{i} \dot{\mathbf{x}}_{i}(v) d v d s .
$$

We can obtain

$$
\begin{aligned}
\dot{V}(t)= & 2 \mathbf{x}_{i}^{T}(t) \mathbf{P}_{i} \dot{\mathbf{x}}_{i}(t) \\
& +2\left[\mathbf{x}_{i}^{T}(t) \mathbf{N}_{i 1}+\mathbf{x}_{i}^{T}\left(b_{i, k} h\right) \mathbf{N}_{2 i}+\dot{\mathbf{x}}_{i}^{T}(t) \mathbf{N}_{i 3}\right] \\
& \cdot\left[\mathbf{x}_{i}(t)-\mathbf{x}_{i}\left(b_{i, k} h\right)-\int_{b_{i, k} h}^{t} \dot{\mathbf{x}}_{i}(s) d s\right] \\
& +2\left[\mathbf{x}_{i}^{T}(t) \mathbf{M}_{i 1}+\mathbf{x}_{i}^{T}\left(b_{i, k} h\right) \mathbf{M}_{2 i}+\dot{\mathbf{x}}_{i}^{T}(t) \mathbf{M}_{i 3}\right] \\
& \cdot\left[-\mathbf{A}_{i} \mathbf{x}_{i}(t)-\mathbf{B}_{i} \mathbf{K}_{i} \mathbf{x}_{i}\left(b_{i, k} h\right)+\dot{\mathbf{x}}_{i}(t)\right] \\
& +\eta \dot{\mathbf{x}}_{i}^{T}(t) \mathbf{T} \dot{\mathbf{x}}_{i}(t)-\int_{t-\eta}^{t} \dot{\mathbf{x}}_{i}^{T}(s) \mathbf{T} \dot{\mathbf{x}}_{i}(s) d s \\
& +\mathbf{e}_{i}^{T}\left(i_{k} h\right) \Phi \mathbf{e}_{i}\left(i_{k} h\right)-\mathbf{e}_{i}^{T}\left(i_{k} h\right) \Phi \mathbf{e}_{i}\left(i_{k} h\right) .
\end{aligned}
$$

Since $\eta(t)=t-b_{i, k} h$, when $t \in\left[b_{i, k} h+\tau_{i, k}, b_{i, k+1} h+\tau_{i, k+1}\right)$, it leads to

$$
-\int_{t-\eta}^{t} \dot{\mathbf{x}}_{i}^{T}(s) \mathbf{T} \dot{\mathbf{x}}_{i}(s) d s \leq-\int_{b_{i, k} h}^{t} \dot{\mathbf{x}}_{i}^{T}(s) \mathbf{T} \dot{\mathbf{x}}_{i}(s) d s .
$$

Based on the transmission scheme (3), for $i_{i, k} h \in\left[b_{i, k} h\right.$, $\left.b_{i, k+1} h\right)$, we have

$$
\begin{gathered}
\mathbf{e}_{i}^{T}\left(i_{i, k} h\right) \Phi \mathbf{e}_{i}\left(i_{i, k} h\right) \leq \sigma_{i} \mathbf{x}_{i}^{T}\left(b_{i, k} h\right) \Phi \mathbf{x}_{i}\left(b_{i, k} h\right), \\
-2\left[\mathbf{x}_{i}^{T}(t) \mathbf{N}_{i 1}+\mathbf{x}_{i}^{T}\left(b_{i, k} h\right) \mathbf{N}_{2 i}+\dot{\mathbf{x}}_{i}^{T}(t) \mathbf{N}_{i 3}\right] \int_{b_{i, k} h}^{t} \dot{\mathbf{x}}_{i}(s) d s \\
\leq \eta\left[\begin{array}{lll}
\mathbf{x}_{i}^{T}(t) & \mathbf{x}_{i}^{T}\left(b_{i, k} h\right) & \left.\dot{\mathbf{x}}_{i}^{T}(t)\right] \mathbf{N}_{i} \mathbf{T}^{-1} \mathbf{N}_{i}^{T}\left[\begin{array}{c}
\mathbf{x}_{i}^{T}(t) \\
\mathbf{x}_{i}^{T}\left(b_{i, k} h\right) \\
\dot{\mathbf{x}}_{i}^{T}(t)
\end{array}\right]
\end{array}\right.
\end{gathered}
$$$$
+\int_{b_{i, k} h}^{t} \dot{\mathbf{x}}_{i}^{T}(s) \mathbf{T} \dot{\mathbf{x}}_{i}(s) d s
$$

Set $\xi_{i}(t)=\left[\begin{array}{llll}\mathbf{x}_{i}^{T}(t) & \mathbf{x}_{i}^{T}\left(b_{i, k} h\right) & \dot{\mathbf{x}}_{i}^{T}(t)\end{array}\right]^{T}$ and $\mathbf{z}_{i}(t)=$ $\left[\xi_{i}^{T}(t) \mathbf{e}_{i}^{T}\left(i_{k} h\right)\right]^{T}$; we have $\dot{V}(t) \leq \mathbf{z}_{i}^{T}(t) \Theta \mathbf{z}_{i}(t)$, where

$$
\begin{aligned}
& \Theta=\left[\begin{array}{cc}
\Omega+\eta \mathbf{N}_{i} \mathbf{T}^{-1} \mathbf{N}_{i}^{T} & \mathbf{0} \\
* & -\Phi
\end{array}\right], \\
& \Omega=\left[\begin{array}{ccc}
\Theta_{i, 11} & \Theta_{i, 12} & \Theta_{i, 13} \\
* & \Theta_{i, 22} & \Theta_{i, 23} \\
* & * & \Theta_{i, 33}
\end{array}\right], \quad \mathbf{N}_{i}^{T}=\left[\begin{array}{c}
\mathbf{N}_{i 1} \\
\mathbf{N}_{i 2} \\
\mathbf{N}_{i 3}
\end{array}\right] \text {. }
\end{aligned}
$$

By Schur complement, (6) is equivalent to $\Theta<0$, and by (6), we have $\dot{V}(t)<0$. This completes the proof.

\section{Transmission Rate and Control Codesign}

For the transmission scheme (3), it is obvious that the communication load and control performance of systems $H$ are determined by the parameters $\sigma_{i}, \Phi$, and $h$. Note how by enlarging $\sigma_{i}$ we reduce transmission to satisfy the communication constraint, but thus we also might degrade the control performance of systems. Hence, $\sigma_{i}$ can be used as a designed parameter to tradeoff between limited communication resource and desired control performance. As a matter of fact, a smaller $\sigma_{i}$ is quite preferable to optimize control performance of systems. But it is well known that the smaller the $\sigma_{i}$ is, the faster the transmission is, and thus it might lead to flow rate exceeding the limit. In this case, control performance of WSNCS might degrade since it is highly sensitive to network performance. Hence, it is an open question how to determine the smallest $\sigma_{i}$ on the premise that the link capacity is satisfied.

Since we only take the control systems associated with forward flow into account in this paper, $T_{i, k}=$ (sample_size) $/\left(b_{i, k+1} h-b_{i, k} h\right)$ represents the traffic flow rate generated by the control system $i \in H$, where sample_size is the size of each transmitted packet and $b_{i, k+1} h-b_{i, k} h$ denotes the transmission period with which the flow is associated with the control system $i$. Let $\widehat{c}_{l}$ and $\vartheta_{l}$ be the average link capacity and the virtual link capacity margin of link $l \in L$. Now, the infimum of $\sigma_{i}$ is derived by using the following optimal methods. 
4.1. Multihop Wireless Sensor Network Case. For wireless multihop sensor networks-based control systems, we provide the following optimization problem:

$$
\begin{array}{cc}
Q_{1}: \min & \sigma_{i}\left(T_{i, k}\right) \\
\text { s.t. } & (6), \\
\sum_{i \in H, f \in F(i) \& f \in F(l)} T_{i, k} \leq \widehat{\mathcal{c}}_{l}-\vartheta_{l} .
\end{array}
$$

Remark 4. From (3), it is obvious that there exists a map relationship between $\sigma_{i}$ and $T_{i, k}$ based on the definition $T_{i, k}$, and this relation is represented by using $\sigma_{i}\left(T_{i, k}\right) . f \in$ $F(i) \& f \in F(l)$ denotes all of the flows that are associated with the control system $i$ and traverse the link $l$, and $\sum_{i \in H, f \in F(i) \& f \in F(l)} T_{i, k} \leq \widehat{\mathcal{c}}_{l}-\vartheta_{l}$ means that the sum of flow rates generated by all of the control systems transmitting information over link $l$ must satisfy the maximum allowable rate $\widehat{c}_{l}-\vartheta_{l}$ of link $l$.

4.2. Star Topology Wireless Sensor Network Case. For the situation of a star topology wireless sensor network as communication medium of WSNCS, the optimization problem is formulated as follows:

$$
\begin{array}{cc}
Q_{2}: & \min \quad \sigma_{i}\left(T_{i, k}\right) \\
\text { s.t. } \quad(6), \\
T_{i, k} \leq \widehat{\mathcal{c}}_{l}-\vartheta_{l} .
\end{array}
$$

Remark 5. As a matter of fact, the optimization problem $Q_{2}$ for the case of star topology is a special situation of optimization problem $Q_{1}$ when only one flow passes through for each link.

Remark 6. The main objective of the focused transmission rate and control codesign is to search the infimums of $\sigma_{i}$ associated with flow rate $T_{i, k}(i \in H)$ for the control systems $H$, and this problem has not been fully investigated in [15-18]. By solving the optimization problems $Q_{1}$ and $Q_{2}$, the infimums of $\sigma_{i}$ for the multihop and star topology networks-based control systems under a specific link capacity constraint can be obtained. Choosing the obtained infimums of $\sigma_{i}$, the optimal control performance can be achieved under the controllers meeting (6). If the $\sigma_{i}$ is set to values that are smaller than the infimums of $\sigma_{i}$, the flow rate will exceed the link capacity constraint due to the too fast transmission based on the transmission scheme (3). Thus this will lead to network performance degradation and not reaching the desired control performance. Hence, the key benefit of the presented optimization problems is to avoid that the $\sigma_{i}$ is given too small such that the systems cannot reach the desired optimal control performance due to flow rate going over the link capacity limits.

Obviously, the problems $Q_{1}$ and $Q_{2}$ are the nontrivial nonlinear interactions posed by the tight coupling relationship between transmission rate and $\sigma_{i}$. To solve this problem, we provide a search algorithm to achieve the optimal solution of the problem $Q_{1}$ and $Q_{2}$.

Algorithm 7.

Step 1. Given scalars $h, \widehat{c}_{l}, \vartheta_{l}(l \in L), k_{\max }$ and feasible matrices $\mathbf{K}_{i}(i \in H)$ meeting (6) when $\sigma_{i}$ are initially set to zero, set $k=0$.

Step 2. For each control system $i$, if there exists a feasible solution satisfying (6), go to Step 3; otherwise, go to Step 6.

Step 3. Execute the transmission scheme (3) and calculate flow rate $T_{i, k}$ generated by the control system $i$.

Step 4. If there exists one control system $i$ that cannot meet constraint condition (15) (or (18)), go to Step 5 in order to update $\sigma_{i}$; otherwise, $k=k+1$. If $k \leq k_{\max }$, go to Step 3; otherwise, go to Step 6.

Step 5. Set $\sigma_{i}=\sigma_{i}+\varsigma_{i}$, where $\varsigma_{i}\left(0<\varsigma_{i}<1\right)$ is step size; go to Step 2.

Step 6. Exit.

More detailed implementation of Algorithm 7 is shown in Figure 3.

Remark 8. $k$ denotes the run time, $k=0,1,2, \ldots, k_{\max }$, and $k_{\max }$ is a threshold of run time. In general, a large $k_{\max }$ is more preferable to obtain the optimal $\sigma_{i}$ at the cost of increasing computational overhead. In addition, for simplifying the computation, the flow rate $T_{i, k}$ during $\left[b_{i, k} h, b_{i, k+1} h\right)$ may be replaced by the average flow rate. Here the average transmission interval $\bar{T}$ is first defined as the ratio of a given run time $\left(k_{\max } * h\right)$ to the number of transmitted samples, and then the average flow rate can be computed by the formula (sample_size) $/ \bar{T}$.

\section{Illustrative Examples}

In this section, we verify the effectiveness of the proposed method by using two examples.

Example 1. In this example, WSNCS consists of a star topology network and one closed-loop control system. The control system is described as [21]

$$
\dot{\mathbf{x}}(t)=\left[\begin{array}{cc}
0 & 1 \\
0 & -0.1
\end{array}\right] \mathbf{x}(t)+\left[\begin{array}{c}
0 \\
0.1
\end{array}\right] \mathbf{u}(t) .
$$

In this simulation, packet_size is 100 bytes, $\widehat{c}_{l}=6000 \mathrm{bps}, \vartheta_{1}=$ $10 \mathrm{bps}, \varsigma_{1}=0.01, k_{\max }=500$, and $\eta=0.8695$, and the initial state is $x_{0}=\left[\begin{array}{ll}0.3 & 0.1\end{array}\right]^{T}$. And in the transmission scheme (3), we set

$$
\Phi=\left[\begin{array}{ll}
1 & 0 \\
0 & 1
\end{array}\right] .
$$

By Monte Carlo method, we produce transmission delay during $[0, \bar{\tau}], \bar{\tau}=2 * h$. We choose $\mathbf{K}=\left[\begin{array}{ll}-3.75 & -11.5\end{array}\right]$ 


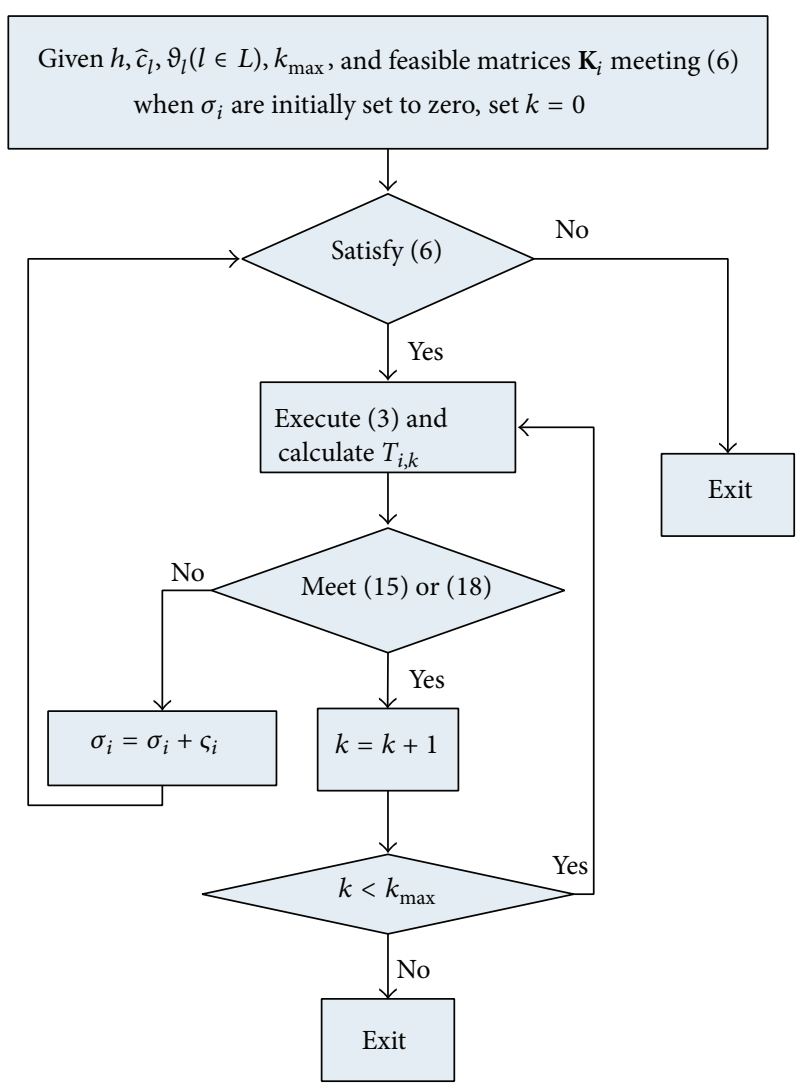

FIGURE 3: Flow chart of the proposed algorithm.

TABLE 1: Comparison with constant sampling.

\begin{tabular}{lccc}
\hline$h$ & $\sigma_{1}$ & $\bar{T}$ & Average flow rate \\
\hline 0.001 & 0.0101 & 0.1817 & 4403 \\
0.05 & 0.0105 & 0.1938 & 4128 \\
0.1 & 0.01 & 0.2604 & 3072 \\
\hline
\end{tabular}

TABLE 2: Comparison with constant sampling.

\begin{tabular}{lccc}
\hline$h$ & $\sigma_{1}$ & $\bar{T}$ & Average flow rate \\
\hline 0.001 & 0.002 & 0.1023 & $7.8201 e+03$ \\
0.05 & 0.01 & 0.1299 & $6.1586 e+03$ \\
0.1 & 0.008 & 0.1086 & $7.3665 e+03$ \\
\hline
\end{tabular}

[21]. By Algorithm 7, the infimums of $\sigma_{1}$ and the average transmission intervals $\bar{T}$ for different sampling period $h$ are obtained and are listed in Table 1. From Table 1, we can find that the flow rate is within the link capacity limit and the average transmission interval $\bar{T}$ is larger than the constant sampling period. This shows that network resource occupation is reduced. Moreover, the data listed in Table 2 show that the flow rate generated by the system exceeds the link capacity constraints when $\sigma_{1}$ is smaller than the infimum.

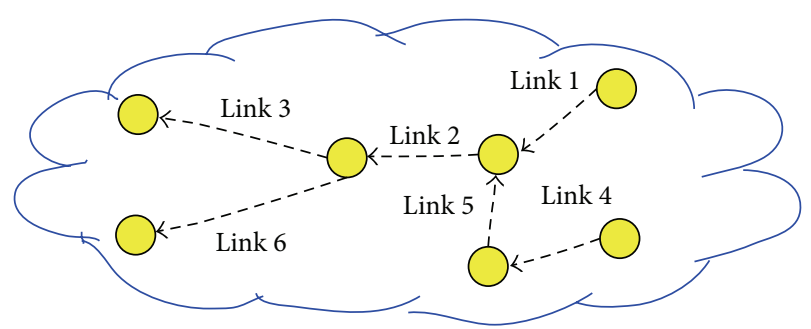

FIGURE 4: A wireless multihop sensor network.

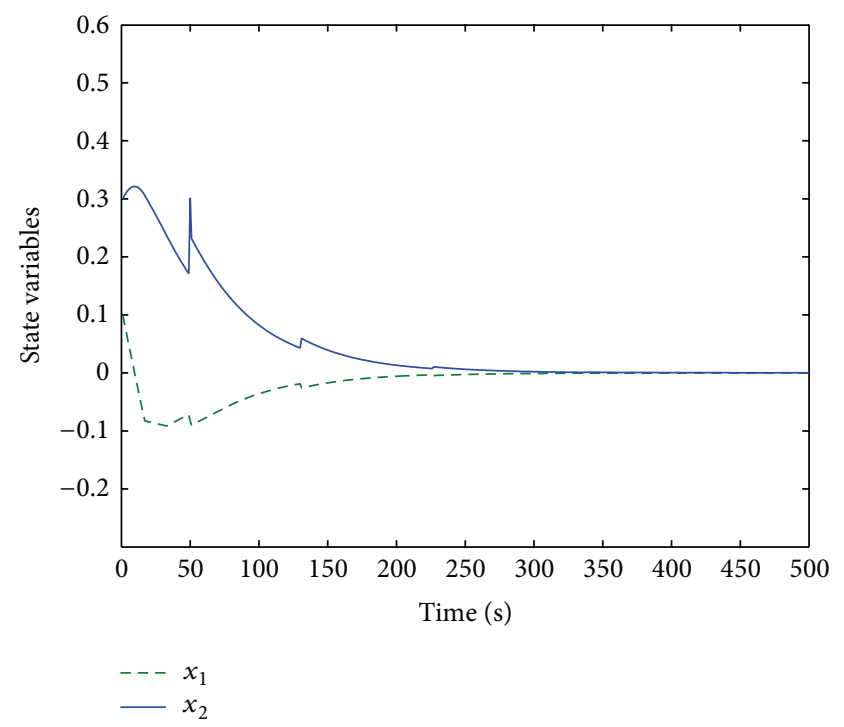

FIGURE 5: The state responses of the system (21).

Example 2. Consider two control systems sharing a wireless multihop sensor network with the parameters (see [21, 22])

$$
\begin{array}{ll}
\mathbf{A}_{1}=\left[\begin{array}{cc}
0 & 1 \\
0 & -0.1
\end{array}\right], \quad \mathbf{B}_{1}=\left[\begin{array}{c}
0 \\
0.1
\end{array}\right], \\
\mathbf{A}_{2}=\left[\begin{array}{ll}
0 & 1 \\
1 & 0
\end{array}\right], & \mathbf{B}_{2}=\left[\begin{array}{l}
0 \\
1
\end{array}\right] .
\end{array}
$$

Similar to Example 1, we set

$$
\Phi=\left[\begin{array}{ll}
1 & 0 \\
0 & 1
\end{array}\right] .
$$

And the topology structure of wireless multihop sensor network is shown in Figure 4. Note that link 2 is shared by the above two plants in the presented topology structure. In addition, some necessary simulation parameters still need to be determined in advance; we set packet_size to 100 bytes, the link capacity $\widehat{c}_{i}=5000 \mathrm{bps}$, virtual link capacity margin $\vartheta_{i}=$ $10 \mathrm{bps}, \varsigma_{i}=0.01(i=1,2), \eta=0.9940$, and $k_{\max }=500$. For the control system (21), we choose the same controller gain and the initial state as those in Example 1, and the controller gain $\mathbf{K}=[-1.006-1.006]$ [22] and the initial state $x_{2}(0)=$ $\left[\begin{array}{ll}0.03 & 0.01\end{array}\right]^{T}$ are utilized for the control system (22). For the sampling period $h=0.05 \mathrm{~s}$, transmission delay that belongs to $[0,2 * h]$ can be produced by Monte Carlo method. 


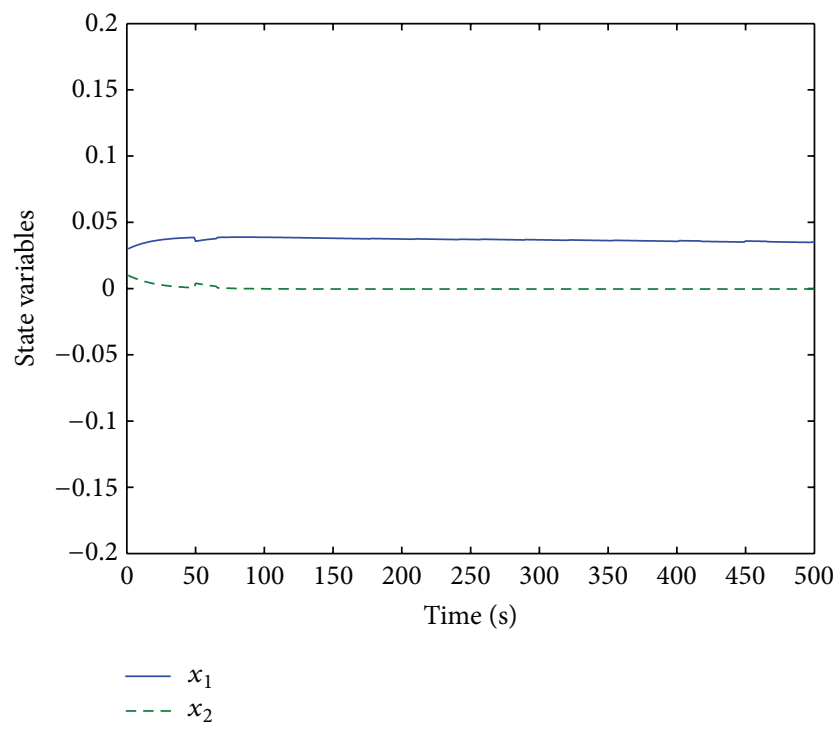

FIGURE 6: The state responses of the system (22).

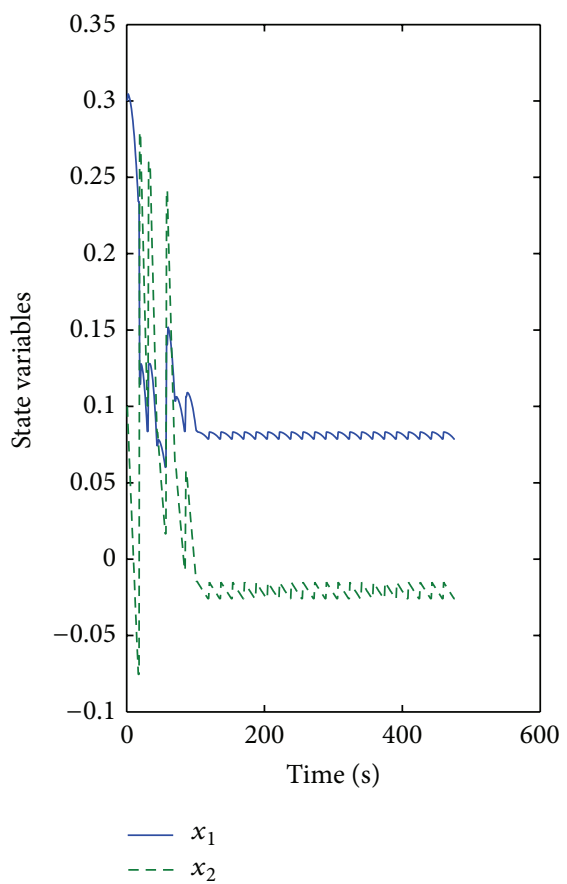

(a)

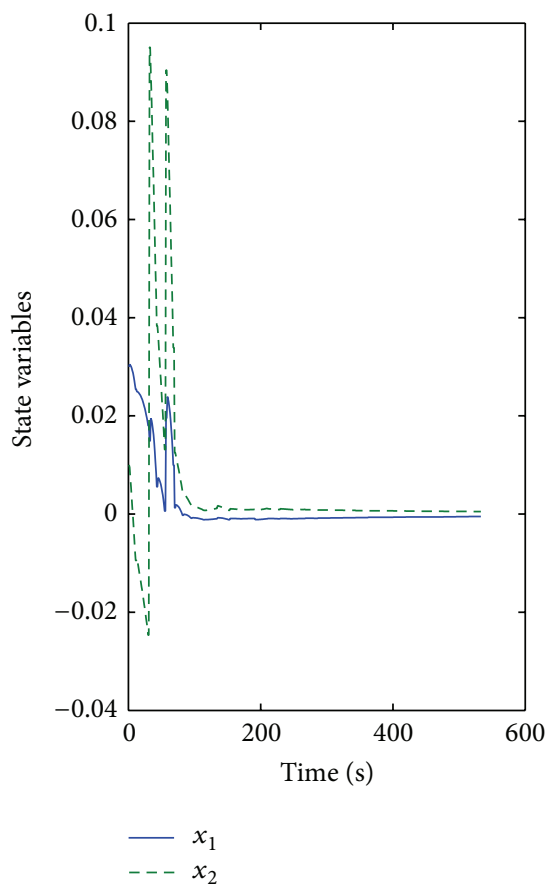

(b)

Figure 7: The state responses of the systems (a) (21) and (b) (22).

By Algorithm 7, the infimums 0.3561 and 0.3565 of $\sigma_{1}$ and $\sigma_{2}$ are obtained, respectively. Figures 5 and 6 show the state responses of the two control systems under the obtained infimums of $\sigma_{i}(i=1,2)$. From Figures 5 and 6, one can easily see that the two systems have been stabilized under the presented controller when performing the transmission scheme (3) if $\sigma_{1}$ and $\sigma_{2}$ are set to 0.3561 and 0.3565 . However, if the infimums of $\sigma_{i}(i=1,2)$ are not searched (see [1518]), the systems that share a network with limited bandwidth may fail to be stabilized when given $\sigma_{i}$ are smaller than the infimums. As shown in Figure 7, the system (21) is unstable when $\sigma_{1}$ is 0.35 , and the system (22) is stabilized with a large overshoot compared with that in Figure 6 under the case of $\sigma_{2}=0.352$. This is because the flow rates exceed the link 
capacity constraint leading to the degradation of network performance when the smaller $\sigma_{i}(i=1,2)$ than the infimums are set.

\section{Conclusions}

Transmission rate and control codesign is investigated for wireless sensor networked control system with time-varying delay. The most appealing advantage of the proposed method is that an optimal event-triggered transmission condition is determined based on the wireless link capacity constraint and asymptotical stability condition. Moreover, the designed method is simple and easily applicable since no model assumptions on the time-varying delay and flow rate are made. The examples and simulations show the effectiveness of the proposed method.

\section{Conflict of Interests}

The authors declare that there is no conflict of interests regarding the publication of this paper.

\section{Acknowledgments}

The authors would like to acknowledge the National Natural Science Foundation of China under Grants 61104093, 61174119, 61034006, 61174026, and 61104003, the Special Program for Key Basic Research founded by MOST under Grant 2010CB334705, the National High Technology Research and Development Program of China (863 Program) under Grant 2011AA040101, the Opening Project of Key Laboratory of Networked Control Systems, Chinese Academy of Sciences, and Scientific Research Project of Liaoning Province of China under Grant L2012141.

\section{References}

[1] Y. Uchimura, "Wireless network based control with time varying delay," in Proceedings of the 34th Annual Conference of the IEEE Industrial Electronics (IECON '08), pp. 2962-2967, Orlando, Fla, USA, November 2008.

[2] A. Flammini, P. Ferrari, D. Marioli, E. Sisinni, and A. Taroni, "Wired and wireless sensor networks for industrial applications," Microelectronics Journal, vol. 40, no. 9, pp. 1322-1336, 2009.

[3] J. Li, Q. Zhang, H. Yu, and M. Cai, "Real-time guaranteed cost control of MIMO networked control systems with packet disordering," Journal of Process Control, vol. 21, no. 6, pp. 967975, 2011.

[4] F. Xia, Y.-C. Tian, Y. Li, and Y. Sun, "Wireless sensor/actuator network design for mobile control applications," Sensors, vol. 7, no. 10, pp. 2157-2173, 2007.

[5] X. Luan, P. Shi, and F. Liu, "Stabilization of networked control systems with random delays," IEEE Transactions on Industrial Electronics, vol. 58, no. 9, pp. 4323-4330, 2011.

[6] N. J. Ploplys, P. A. Kawka, and A. G. Alleyne, "Closed-loop control over wireless networks," IEEE Control Systems Magazine, vol. 24 , no. 3 , pp. 58-71, 2004.
[7] A. Laprovitta, G. Peretti, and E. Romero, "Applying the analog configurability test approach in a wireless sensor network application," Journal of Electrical and Computer Engineering, vol. 2014, Article ID 309193, 14 pages, 2014.

[8] X. L. Zhang, W. H. Zhang, X. R. Zhang, Z. Z. Xu, and F. Zhang, "Optimized deployment of cluster head nodes in wireless network for the greenhouse," in Proceedings of the International Conference on Wireless Communications, Networking and Mobile Computing (WiCOM '08), pp. 1-5, Dalian, China, October 2008.

[9] M. Tabbara, A. Rantzer, and D. Nešić, "On controller \& capacity allocation co-design for networked control systems," Systems and Control Letters, vol. 58, no. 9, pp. 672-676, 2009.

[10] H. Rehbinder and M. Sanfridson, "Scheduling of a limited communication channel for optimal control," Automatica, vol. 40, no. 3, pp. 491-500, 2004.

[11] S.-L. Dai, H. Lin, and S. S. Ge, "Scheduling-and-control codesign for a collection of networked control systems with uncertain delays," IEEE Transactions on Control Systems Technology, vol. 18, no. 1, pp. 66-78, 2010.

[12] Y. Jin and G. W. Bai, "Energy-aware adaptive cooperative FEC protocol in MIMO channel for wireless sensor networks," Journal of Electrical and Computer Engineering, vol. 2013, Article ID 891429, 9 pages, 2013.

[13] O. Jerew, K. Blackmore, and W. F. Liang, "Mobile base station and clustering to maximize network lifetime in wireless sensor networks," Journal of Electrical and Computer Engineering, vol. 2012, Article ID 902862, 13 pages, 2012.

[14] W. P. M. H. Heemels, R. J. A. Gorter, A. van Zijl et al., "Asynchronous measurement and control: a case study on motor synchronization," Control Engineering Practice, vol. 7, no. 12, pp. 1467-1482, 1999.

[15] P. Tabuada, "Event-triggered real-time scheduling of stabilizing control tasks," IEEE Transactions on Automatic Control, vol. 52, no. 9, pp. 1680-1685, 2007.

[16] X. Wang and M. D. Lemmon, "Event-triggering in distributed networked control systems," IEEE Transactions on Automatic Control, vol. 56, no. 3, pp. 586-601, 2011.

[17] M. C. F. Donkers and W. P. M. H. Heemels, "Output-based event-triggered control with guaranteed $L_{\infty}$ gain and improved and decentralised event triggering," IEEE Transactions on Automatic Control, vol. 57, no. 6, pp. 1362-1376, 2012.

[18] C. Peng and T. C. Yang, "Event-triggered communication and $H_{\infty}$ control co-design for networked control systems," Automatica, vol. 49, no. 5, pp. 1326-1332, 2013.

[19] H. Yu and P. J. Antsaklis, "Event-triggered output feedback control for networked control systems using passivity: achieving $L_{2}$ stability in the presence of communication delays and signal quantization," Automatica, vol. 49, no. 1, pp. 30-38, 2013.

[20] J. Bai, E. Eyisi, F. Qiu, Y. Xue, and X. D. Koutsoukos, "Optimal cross-layer design of sampling rate adaptation and network scheduling for wireless networked control systems," in Proceedings of the 3rd IEEE/ACM International Conference on CyberPhysical Systems, pp. 107-116, Washington, DC, USA, April 2012.

[21] D. Yue, Q.-L. Han, and C. Peng, "State feedback controller design of networked control systems," IEEE Transactions on Circuits and Systems II: Express Briefs, vol. 51, no. 11, pp. 640644, 2004.

[22] B. Tang, G.-P. Liu, and W.-H. Gui, "Improvement of state feedback controller design for networked control systems," IEEE Transactions on Circuits and Systems II: Express Briefs, vol. 55, no. 5, pp. 464-468, 2008. 

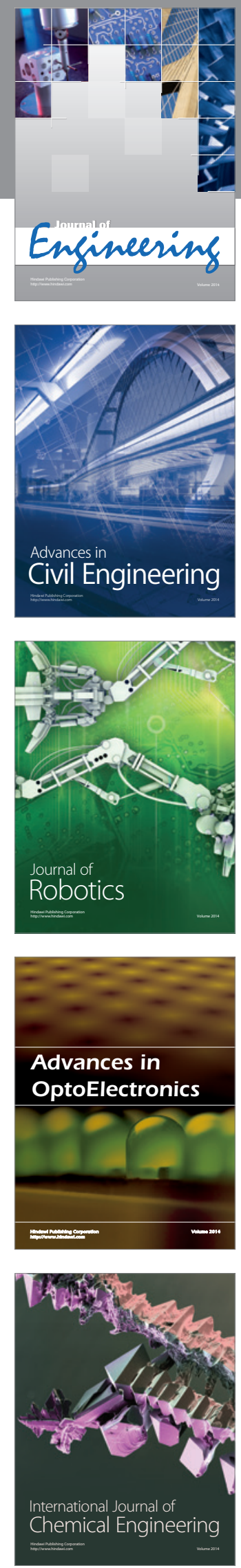

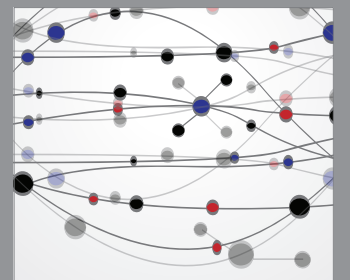

The Scientific World Journal
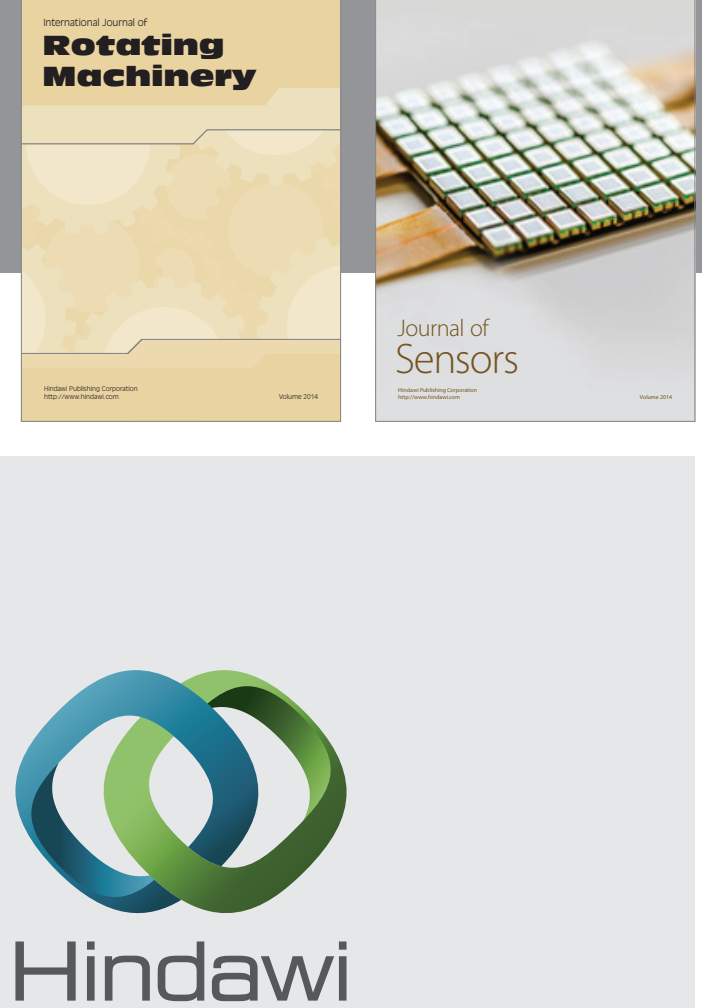

Submit your manuscripts at http://www.hindawi.com
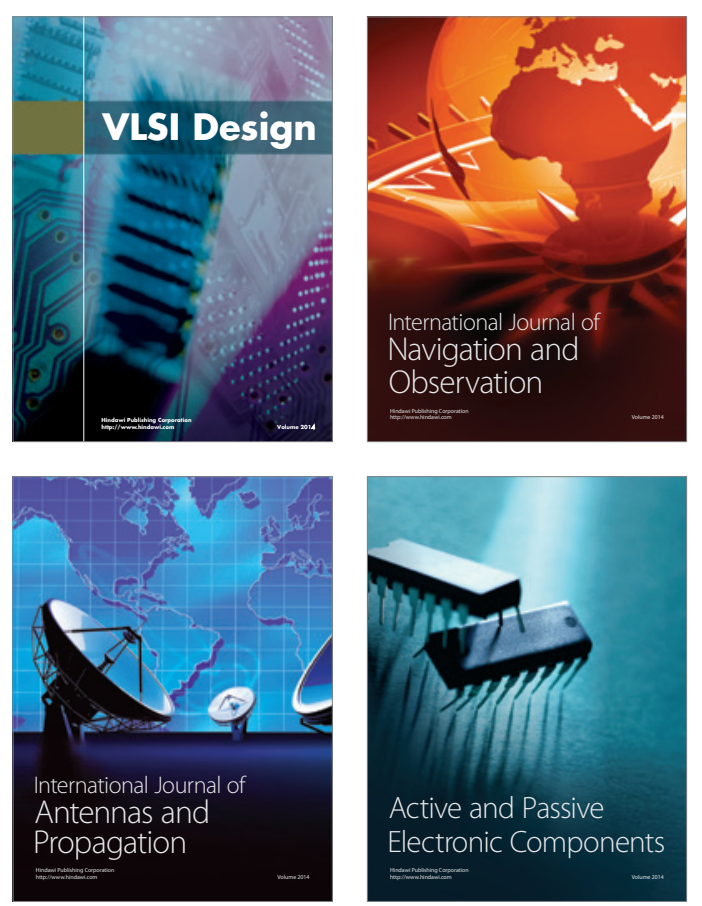
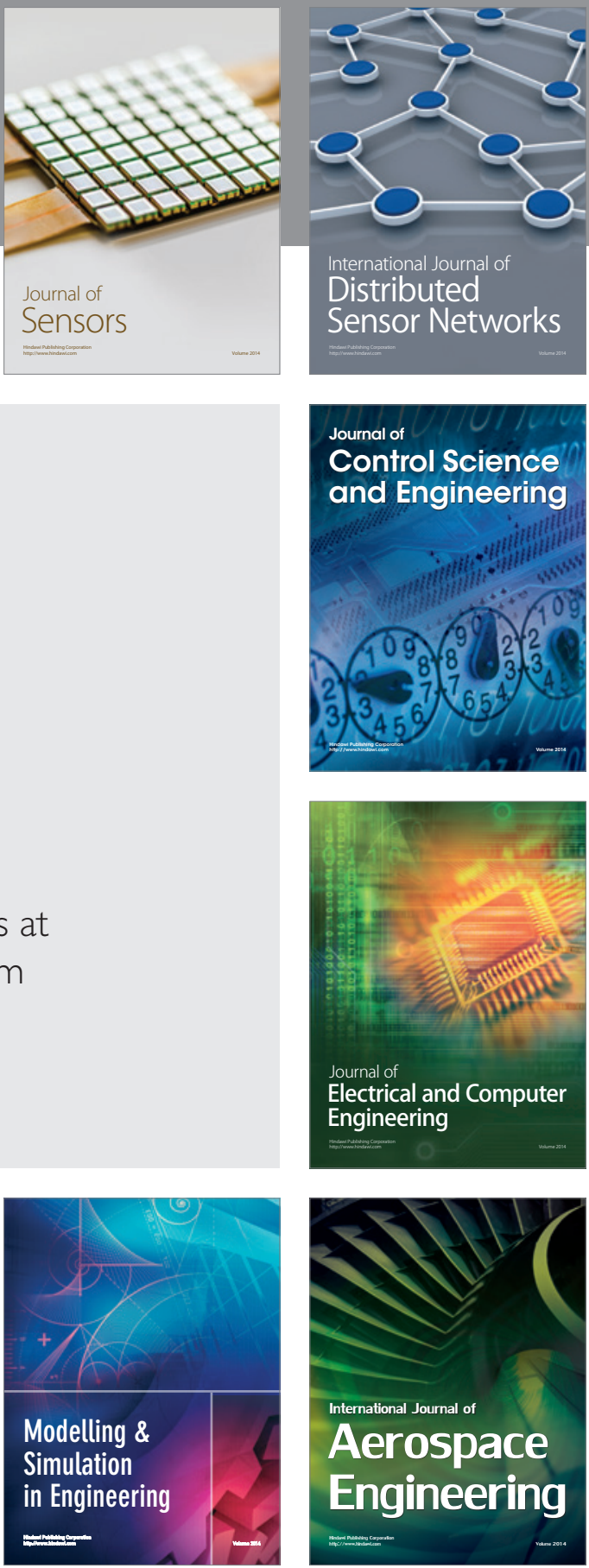

Journal of

Control Science

and Engineering
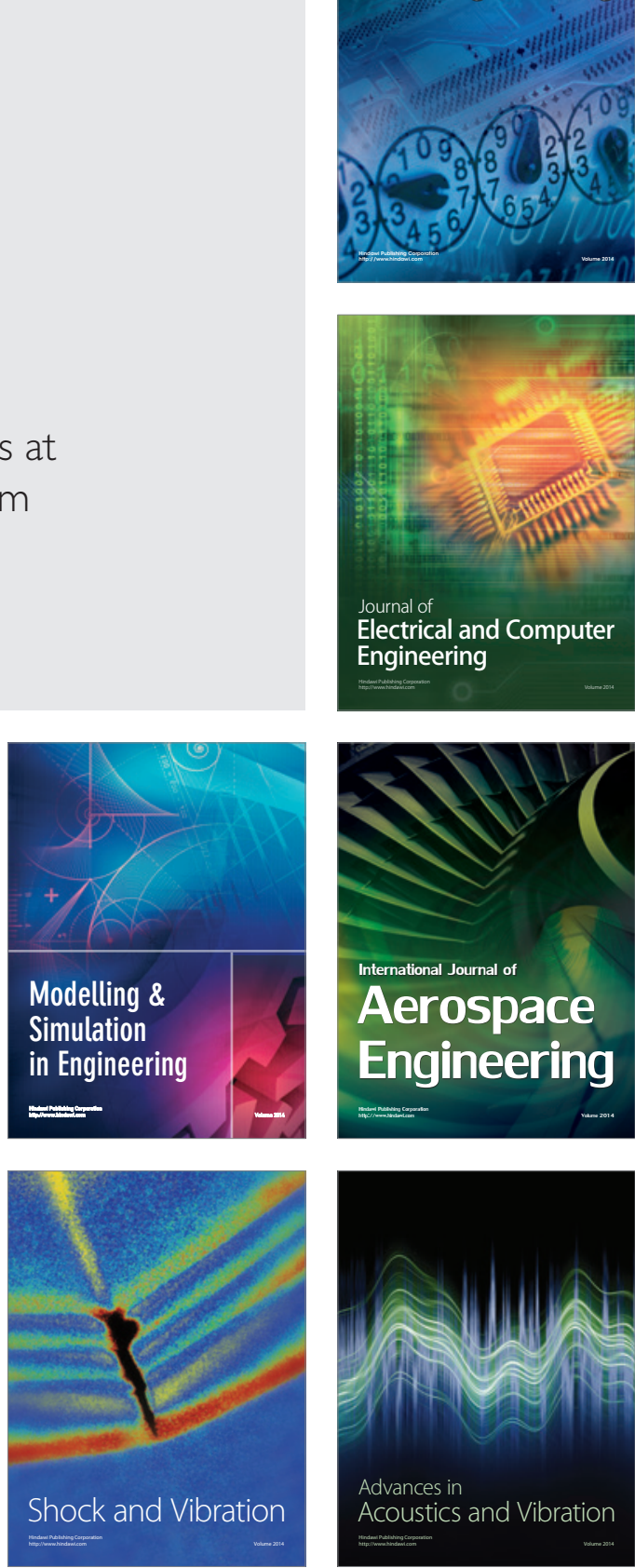